\title{
Clinical efficacy and safety of palliative esophageal stenting without fluoroscopy: a systematic review and meta-analysis
}

\section{다 (1) $(2)$}

Authors

Saurabh Chandan*, 1, Babu P. Mohan*,2, Shahab R. Khan², Neil Bhogal'1, Andrew Canakis³, Mohammad Bilal'5, Amaninder S. Dhaliwal ${ }^{1}$, Muhammad Aziz ${ }^{6}$, Harmeet S. Mashiana ${ }^{1}$, Shailender Singh ${ }^{1}$, Wade Lee-Smith ${ }^{7}$, Suresh Ponnada ${ }^{4}$, Ishfaq Bhat ${ }^{1}$, Douglas Pleskow ${ }^{5}$

Institutions

1 Gastroenterology and Hepatology, University of Nebraska Medical Center, Omaha, Nebraska, United States

2 Internal Medicine, University of Arizona, Banner University Medical Center, Tucson, Arizona, United States

3 Boston University Medical Center, Boston, Massachusetts, United States

4 Internal Medicine, Carilion Roanoke Memorial Hospital, Roanoke, Virginia, United States

5 Gastroenterology \& Hepatology, Beth Israel Deaconess Medical Center, Harvard Medical School, Boston, Massachusetts, United States

6 Internal Medicine, University of Toledo, Ohio, United States

7 University Library, University of Toledo, Ohio, United States

submitted 6.2.2020

accepted after revision 30.3 .2020

Bibliography

DOI https://doi.org/10.1055/a-1164-6398 |

Endoscopy International Open 2020; 08: E944-E952

(c) Georg Thieme Verlag KG Stuttgart · New York eISSN 2196-9736

Corresponding author

Saurabh Chandan, MD, Department of Gastroenterology \& Hepatology, 982000 University of Nebraska Medical Center, Omaha, NE 68198, United States

Fax: +1-402-559-9004

saurabhchandan@gmail.com

\section{ABSTRACT}

Background and study aims Despite advances in curative treatments for esophageal cancer, many patients often present with advanced disease. Dysphagia resulting in significant weight loss and malnutrition leads to poor quality of life. Palliative esophageal stenting with self-expanding metal stents (SEMS) helps alleviate symptoms and prolongs survival. However, access to fluoroscopy may be limited at certain centers causing delay in patient care.

Methods We searched multiple databases from inception to November 2019 to identify studies evaluating the efficacy and safety of endoscopic palliative esophageal stenting and selected only those studies where fluoroscopic guidance was not used. Our primary aim was to calculate the overall technical as well as clinical success. Using meta-regression analysis, we also evaluated the effect of tumor location and obstruction length on overall technical and clinical success.

Results A total of 1778 patients from 17 studies were analyzed. A total of 2036 stents were placed without the aid of fluoroscopy. The pooled rate of technical success was 94.7\% (Cl 89.9-97.3, PI 55-99; $\left.I^{2}=85\right)$ and clinical success was $82.1 \%\left(\mathrm{Cl} 67.1-91.2\right.$, PI 24-99; $\left.\mathrm{I}^{2}=87\right)$. Based on metaregression analysis both the length of obstruction and tumor location did not have any statistically significant effect on technical and clinical success. The pooled rate of adverse events was $4.1 \%\left(\mathrm{Cl} 2.4-7.2 ; I^{2}=72\right)$ for stent migration, $8.1 \%\left(\mathrm{Cl} 4.1-15.4 ; \mathrm{I}^{2}=89\right)$ for tumor overgrowth and $1.2 \%$ $\left(\mathrm{Cl} 0.7-2 ; \mathrm{I}^{2}=0\right)$ for perforation. The most frequent clinical adverse event was retro-sternal chest pain.

Conclusion Palliative esophageal stenting without fluoroscopy using SEMS is both safe and effective in patients with advanced esophageal cancer.

Supplementary material: Supplementary Fig. S1-4, Appendix A+B, Supplementary Table S1

Online content viewable at:

https://doi.org/10.1055/a-1164-6398

* These authors contributed equally. 


\section{Introduction}

It is estimated that 17,650 cases of esophageal cancer will be diagnosed each year, and 16,080 deaths are expected from the disease in the United States [1]. Globally, of the estimated 456,000 cases of esophageal cancer diagnosed in 2012, 398,000 were squamous cell carcinomas (SCC) and 52,000 were adenocarcinomas [2]. Incidence rates for adenocarcinoma of the esophagus have been increasing dramatically, primarily owing to increases in known risk factors such as obesity [3]. In the United States in particular, as well as in several western countries, smoking and excessive alcohol consumption account for approximately $90 \%$ of the total cases of esophageal squamous cell carcinoma (SCC) [4].

Patients with esophageal cancer present with difficulty swallowing or dysphagia and associated weight loss caused by obstruction of the esophagus by the tumor. Progressive dysphagia usually occurs once the esophageal lumen diameter is less than $13 \mathrm{~mm}$, which indicates advanced disease and is the predominant symptom in more than $70 \%$ of patients [5]. The primary goal of esophageal stenting in patients with advanced disease is to relieve dysphagia and thereby help in preventing worsening malnutrition. Compared with parenteral nutrition, endoscopic stent placement significantly improves a patient's quality of life by restoring the ability to take in food and fluids orally. Despite advances in diagnosis and treatment, the 5-year survival rate for all patients diagnosed with esophageal cancer remains dismal, ranging from $15 \%$ to $20 \%$ [6].

Self-expanding metal stents (SEMS) have been used for palliation of malignant dysphagia since the early 1990 s [7], however, use of fluoroscopy for this purpose can be time-consuming, expose patients to unnecessary radiation, and can occasionally be inaccurate [8]. Access to fluoroscopic services may also not be readily available in certain medical centers. Traditionally, SEMS have been placed under direct endoscopic visualization, however, more recently, a through-the-scope technique has also been described [9]. The aim of our study was to evaluate the overall clinical efficacy and safety of esophageal SEMS placement without the aid of fluoroscopy.

\section{Methods}

\section{Search strategy}

We conducted a comprehensive search of several databases from inception to December, 2019. The databases included Ovid MEDLINE and Epub Ahead of Print, In-Process and other non-indexed citations, Ovid Embase, Ovid Cochrane Central Register of Controlled trials, Ovid Cochrane Database of Systematic Reviews, Web of Science Core Collection and Scopus. Two experienced medical librarians, using inputs from the study authors, helped with the literature search. Controlled vocabulary supplemented with keywords was used to search for studies of interest. In our search strategy we included fluoroscopy along with phrases associated with the procedure such as direct endoscopic visualization, direct endoscopic placement etc. to maximize our literature search. The full search strategy is available in Supplementary Appendix-A. The PRISMA
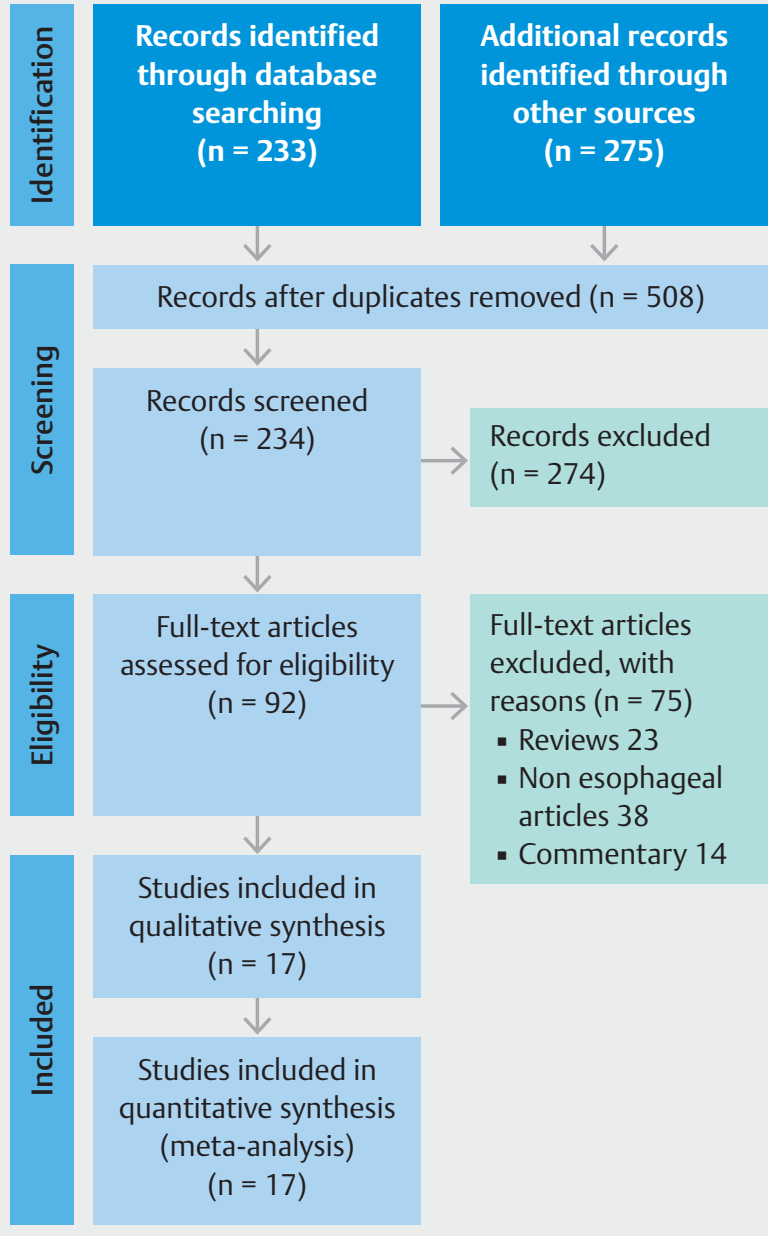

Full-text articles assessed for eligibility $(n=92)$

Full-text articles excluded, with reasons $(n=75)$

- Reviews 23

- Non esophageal articles 38

- Commentary 14

\section{Studies included in}

qualitative synthesis

$$
(n=17)
$$

Studies included in quantitative synthesis (meta-analysis) $(n=17)$

- Fig. 1 Study selection flow chart/PRISMA. From: Moher D, Liberati A, Tetzlaff J, Altman DG, The PRISMA Group (2009). Preferred Reporting Items for Systematic Reviews and Meta-Analyses: The PRISMA Statement. PLoS Med 6(7): e1000097. doi:10.1371/journal. pmed 1000097

and MOOSE checklists were followed as appropriate and are provided in - Fig. 1 and Supplementary Appendix B [10,11]. Reference lists of evaluated studies were examined to identify other studies of interest.

\section{Study selection}

In this meta-analysis, we included studies that evaluated the clinical outcomes of palliative esophageal stent placement without fluoroscopy. Studies were included irrespective of the study sample-size, inpatient/ outpatient setting, and geography as long as they provided data needed for the analysis.

Studies done in the pediatric population (Age < 18 years), and studies not published in English language were our only exclusion criteria. In case of multiple publications from the same cohort and/or overlapping cohorts, data from the most recent and/or most appropriate comprehensive report were retained. When needed, authors were contacted via email for clarification of data and/or study-cohort overlap. The retained studies 
were decided by two authors (B.P.M., S.C.) based on the publication timing (most recent) and/or the sample size of the study (largest).

\section{Data abstraction and quality assessment}

Data on study-related outcomes in the individual studies were abstracted onto a standardized form by at least two authors (SC, SRK), and two authors (BPM, SC) did the quality scoring independently.

The Newcastle-Ottawa scale for cohort studies was used to assess the quality of studies [12]. This quality score consisted of 8 questions, the details of which are provided in $>$ Table 1.

\section{Outcomes assessed}

Outcomes assessed were: 1) pooled rate of overall technical success, as defined by successful deployment of the esophageal stent; 2) pooled rate of clinical success, as defined by improvement in post procedure dysphagia; and 3) pooled rate of most frequently reported stent related adverse events (AEs), including stent migration, tumor overgrowth and perforation.

Pre-determined meta-regression analysis were planned to evaluate the effect of tumor location (proximal, mid or distal esophagus) and obstruction length on overall technical and clinical success.

\section{Statistical analysis}

We used meta-analysis techniques to calculate the pooled estimates in each case following the methods suggested by DerSimonian and Laird using the random-effects model [13]. When incidence of an outcome was zero in a study, a continuity correction of 0.5 was added to the number of incident cases before statistical analysis [14]. We assessed heterogeneity between study-specific estimates by using Cochran Q statistical test for heterogeneity, 95\% prediction interval (PI), which deals with the dispersion of the effects [15-17] and the $\mathrm{I}^{2}$ statistics [18, 19]. In this, values less than $30 \%, 30 \%$ to $60 \%, 61 \%$ to $75 \%$, and greater than $75 \%$ were suggestive of low, moderate, substantial, and considerable heterogeneity, respectively [20]. Publication bias was ascertained, qualitatively, by visual inspection of funnel plot and quantitatively, by the Egger test [21]. When publication bias was present, further statistics using the fail-Safe N test and Duval and Tweedie's 'Trim and Fill' test was used to ascertain the impact of the bias [22]. Three levels of impact were reported based on the concordance between the reported results and the actual estimate if there were no bias. The impact was reported as minimal if both versions were estimated to be the same, modest if effect size changed substantially but the final finding would still remain the same, and severe if basic final conclusion of the analysis is threatened by the bias [23]. $P \geq 0.05$ was used a-priori to define the significance of difference between the groups compared as provided by the statistical software.

All analyses were performed using Comprehensive Meta-Analysis (CMA) software, version 3 (BioStat, Englewood, New Jersey).

\section{Results}

\section{Search results and population characteristics}

From an initial 783 studies, 234 records were screened after removal of duplicates. Ninety-two full-length articles were assessed and 17 studies were included in the final analysis that reported on the outcomes of palliative esophageal stenting without the aid of fluoroscopy.

In 14 studies, SEMS deployment was performed under direct endoscopic vision whereas in two studies, the stent was deployed without the aid of endoscopic visualization. In one study, through-the-scope (TTS) stent deployment method was used. Pre-insertion dilation was performed using Savory and CRE Balloon dilators. The most commonly used esophageal stent was Ultraflex (Boston Scientific, Marlborough Massachusetts, United States). Population characteristics including type/length of stent used are described in Supplementary Table S1. Details on the type of instruments used and whether or not the instrument was successfully able to traverse the stenosis are described in $>$ Table 2 . In two studies, the stenosis was traversed successfully without prior dilation [24,25].

\section{Characteristics and quality of included studies}

There were no multicenter or population-based studies. Overall, four studies were low quality, twelve studies were considered to be of medium quality and one study was of high quality. Majority of the studies were single center observational studies. As a result of this, the MOOSE checklist was followed and is presented as Supplementary Appendix-B.

\section{Meta-analysis outcomes}

A total of 1778 patients were included in the analysis from 17 studies. In all, 2036 esophageal self-expanding metal stents (SEMS) were placed.

The pooled rate of technical success was $94.7 \%(95 \% \mathrm{Cl}$ 89.9-97.3) ( $\triangleright$ Fig.2) and the pooled rate of clinical success was $82.1 \%$ (95\% Cl 67.1-91.2) (\ Fig.3). Most frequently reported AEs were stent migration, tumor overgrowth and perforation. The pooled rate of stent migration was $4.1 \%(95 \% \mathrm{Cl}$ 2.4-7.2), tumor overgrowth was $8.1 \%$ (95\% Cl 4.1-15.4), and perforation was $1.2 \%$ (95\% Cl 0.7-2) (Supplementary Fig. S1, Supplementary Fig. S2, Supplementary Fig.S3). Of the perforations in seven patients, five were intra-procedural related to stent insertion and two were during balloon dilation prior to stent insertion. The $12 \%$ heterogeneity along with the $95 \%$ prediction intervals for the corresponding pooled rates are summarized in $>$ Table 3 .

\section{Meta-regression}

Meta-regression analysis was done to assess the predictive effects of tumor location and tumor obstruction length on the outcomes of interest. The software uses the Knapp-Hartung method, where $P<0.05$ is considered significant and would indicate a potentially possible predictive effect. The results of tumor location on technical success were as follows: upper third $P=0.6$; mid third $P=0.2$, and lower third $P=0.2$. The results of tumor location on clinical success were as follows: up- 


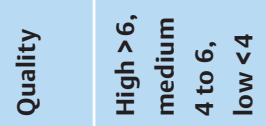

菅

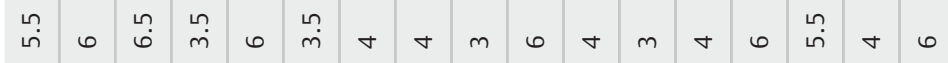

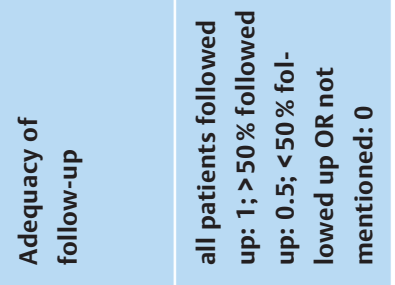

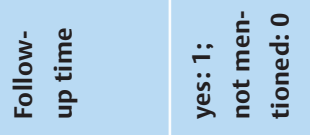

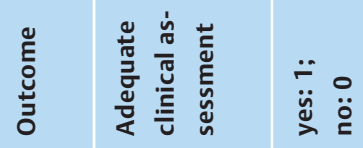

Illin:

min

III

in in

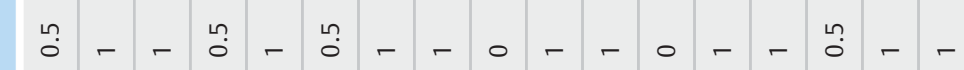

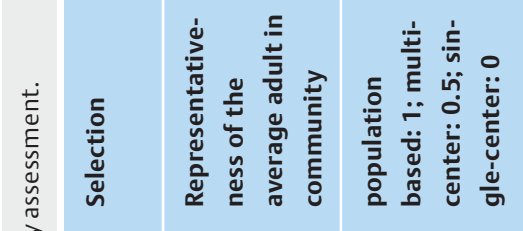

$\begin{array}{lllllllllllllllllllll}n & 0 & 0 & 0 & 0 & 0 & 0 & 0 & 0 & 0 & 0 & 0 & 0 & 0 & 0 & 0 & 0\end{array}$

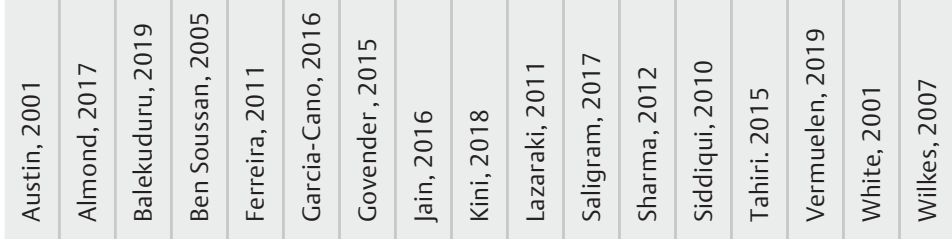


- Table 2 Details of instruments used, scope passage.

\begin{tabular}{|c|c|c|c|}
\hline \multirow[t]{2}{*}{ Study } & \multirow[t]{2}{*}{ Instrument type } & \multicolumn{2}{|c|}{ Pre-dilation Scope passed } \\
\hline & & Yes & No \\
\hline Austin, 2001 & Olympus XQ200, Keymed, Southend of Sea, UK) & $x$ & - \\
\hline Almond, 2017 & NR & $x$ & $x$ \\
\hline Balekuduru, 2019 & 180 GIF180 (Olympus, Tokyo, Japan) & - & $x$ \\
\hline Ben Soussan, 2005 & Olympus XP 160; 5.9 mm diameter, Olympus XP20;8.5 mm & $\mathrm{x}$ & $x$ \\
\hline Ferreira, 2011 & Olympus GIF-XP 160; 5.9 mm & $\mathrm{x}$ & $x$ \\
\hline Garcia-Cano, 2016 & Pentax EG-1870 K; Pentax Corporation, Tokyo, Japan, 6 mm & $\mathrm{x}$ & - \\
\hline Govender, 2015 & NR & $\mathrm{x}$ & $\mathrm{x}$ \\
\hline Jain, 2016 & Adult endoscope, Pediatric flexible gastroscope & $\mathrm{x}$ & $\mathrm{x}$ \\
\hline Kini, 2018 & NR & $x$ & $x$ \\
\hline Lazaraki, 2011 & Fujinon EG-250WR5, Fujinon Corporation, Saitama, Japan, 9.4 mm & $x$ & $x$ \\
\hline Saligram, 2017 & Adult endoscope, Pediatric Flexible Gastroscope & $x$ & $x$ \\
\hline Sharma, 2012 & Olympus EVIS 130 Gastroscope & $\mathrm{x}$ & $x$ \\
\hline Siddiqui, 2010 & NR & - & $x$ \\
\hline Tahiri, 2015 & Adult/Pediatric flexible esophagogastroscope & $x$ & $x$ \\
\hline Vermuelen, 2019 & NA & NA & NA \\
\hline White, 2001 & NR & - & $x$ \\
\hline Wilkes, 2007 & Conventional endoscope, Narrow-bore endoscope & $x$ & $x$ \\
\hline
\end{tabular}

\begin{tabular}{lccc} 
Study name & \multicolumn{3}{c}{ Statistics for each study } \\
& Event rate & Lower limit & Upper limit \\
\hline Austin, 2001 & 0.767 & 0.585 & 0.884 \\
Almond, 2017 & 0.997 & 0.981 & 1.000 \\
Balekuduru, 2019 & 0.994 & 0.907 & 1.000 \\
Ben Soussan, 2005 & 0.909 & 0.753 & 0.970 \\
Ferreira, 2011 & 0.983 & 0.891 & 0.998 \\
Garcia-Cano, 2016 & 0.986 & 0.813 & 0.999 \\
Govender, 2015 & 0.905 & 0.874 & 0.929 \\
Jain, 2016 & 0.995 & 0.932 & 1.000 \\
Kini, 2018 & 0.971 & 0.664 & 0.998 \\
Lazaraki, 2011 & 0.933 & 0.858 & 0.969 \\
Saligram, 2017 & 0.953 & 0.910 & 0.977 \\
Sharma, 2012 & 0.923 & 0.609 & 0.989 \\
Siddiqui, 2010 & 0.938 & 0.858 & 0.974 \\
Tahiri, 2015 & 0.990 & 0.854 & 0.999 \\
Vermuelen, 2019 & 0.313 & 0.177 & 0.490 \\
White, 2001 & 0.993 & 0.897 & 1.000 \\
Wilkes, 2007 & 0.918 & 0.845 & 0.959 \\
& 0.947 & 0.899 & 0.973
\end{tabular}

Event rate and $95 \% \mathrm{Cl}$

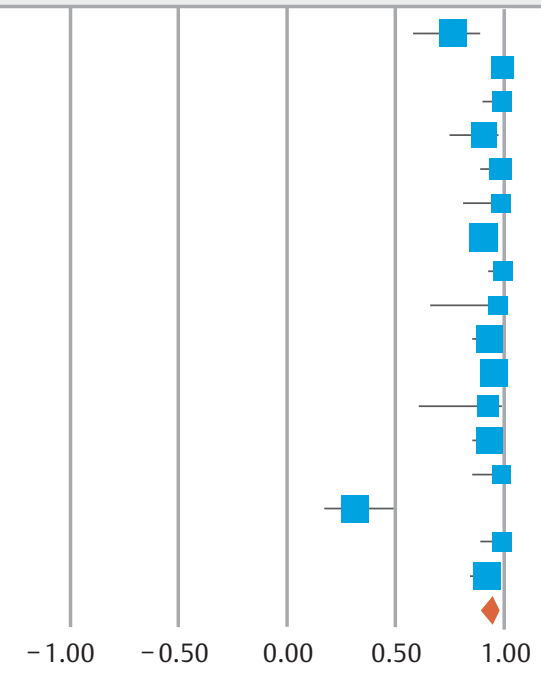

$\checkmark$ Fig. 2 Forest plot, technical success.

per third $P=0.5$, mid third $P=0.4$, and lower third $P=0.2$. Effect of obstruction length on technical success was $P=0.9$ and on clinical success was $P=0.7$. Overall, tumor location and obstruction length did not affect overall technical and clinical success. 


\begin{tabular}{|c|c|c|c|c|c|c|c|c|}
\hline \multirow[t]{2}{*}{ Study name } & \multicolumn{3}{|c|}{ Statistics for each study } & \multicolumn{4}{|c|}{ Event rate and $95 \% \mathrm{Cl}$} & \\
\hline & Event rate & Lower limit & Upper limit & & & & & \\
\hline Austin, 2001 & 0.767 & 0.585 & 0.884 & & & & & \\
\hline Balekuduru, 2019 & 0.852 & 0.740 & 0.921 & & & & & \\
\hline Ben Soussan, 2005 & 0.909 & 0.753 & 0.970 & & & & & \\
\hline Lazaraki, 2011 & 0.988 & 0.919 & 0.998 & & & & & \\
\hline Sharma, 2012 & 0.964 & 0.616 & 0.998 & & & & & \\
\hline Vermuelen, 2019 & 0.857 & 0.676 & 0.945 & & & & & \\
\hline White, 2001 & 0.457 & 0.345 & 0.574 & & & & & \\
\hline \multirow[t]{3}{*}{ Wilkes, 2007} & 0.582 & 0.482 & 0.675 & & & & & \\
\hline & 0.821 & 0.671 & 0.912 & & & & & \\
\hline & & & & -1.00 & -0.50 & 0.00 & 0.50 & 1.00 \\
\hline
\end{tabular}

- Fig. 3 Forest plot, clinical success.

- Table 3 Summary of pooled rates with $\mathrm{I}^{2}, \mathrm{Cl}$ and $\mathrm{PI}$.

\begin{tabular}{|l|l|l|}
\hline & Pooled rate; $95 \%$ confidence interval (CI) & $\mathbf{I}^{\mathbf{2}}$ heterogeneity; 95\% prediction interval (PI) \\
\hline Technical success & $\begin{array}{l}94.7 \%(89.9-97.3) \\
17 \text { studies }\end{array}$ & $\begin{array}{l}85 \% \\
55 \text { to } 99\end{array}$ \\
\hline Clinical success & $82.1 \%(67.1-91.2)$ & $87 \%$ \\
\hline Stent migration & 8 studies & 24 to 99 \\
\hline Tumor overgrowth & $4.1 \%(2.4-7.2)$ & $72 \%$ \\
\hline Perforation & 14 studies & 1 to 22 \\
\hline & $8.1 \%(4.1-15.4)$ & $89 \%$ \\
\hline & 13 studies & 1 to 56 \\
\hline & $1.2 \%(0.7-2)$ & $0 \%$ \\
\hline
\end{tabular}

\section{Validation of meta-analysis results}

\section{Sensitivity analysis}

To assess whether any one study had a dominant effect on the meta-analysis, we excluded one study at a time and analyzed its effect on the main summary estimate. In this analysis, no single study significantly affected the outcome or the heterogeneity.

\section{Heterogeneity}

We assessed dispersion of the calculated rates using the prediction interval $(\mathrm{PI})$ and $\mathrm{I}^{2}$ percentage values. The $\mathrm{PI}$ gives an idea of the range of the dispersion and $\mathrm{I}^{2}$ tell us what proportion of the dispersion is true vs chance [17]. The calculated PIs are reported with the pooled results in Supplementary Table S1. The PI was 55 to 99 for technical success with considerable I $2 \%$. The PI for clinical success was 24 to 99 with considerable $\mathrm{I}^{2} \%$. The PI for stent migration was 1 to 22 with significant $12 \%$, for tumor overgrowth was 1 to 56 with considerable $\mathrm{I}^{2} \%$ and for perforation was 1 to 2 with zero $1^{2} \%$. The results of meta-regression analysis based on obstruction length and tumor location demonstrate that the observed heterogeneity is not explained based on these variables.

\section{Publication bias}

Based on visual inspection of the funnel plot there seems to be presence of publication bias, as the studies are not uniformly distributed across the mean axis. However, based on the quantitative assessment by Eggers regression test, the one-tailed pvalue was 0.05 and the 2 -tailed p-value was 0.1 (Funnel plot: Supplementary Fig. S4). Based on these values, we believe there is evidence for publication bias.

\section{Quality of evidence}

The quality of evidence was rated for results from the meta-analysis according to the GRADE working group approach [26]. Observational studies begin with a low-quality rating and based on the risk of bias, heterogeneity, and publication bias, the quality of this meta-analysis would be considered as low-quality evidence.

\section{Discussion}

Our study demonstrates that palliative esophageal stenting can be both successfully and safely performed without the aid of fluoroscopy. We report a pooled technical success rate of 
$94.7 \%$ and a pooled clinical success rate of $82.1 \%$, derived from 17 studies that evaluated 1778 patients. To the best of our knowledge, this is the first study to report pooled outcomes of esophageal stenting without the aid of fluoroscopy. A recent review by Anderloni et al stated that conventional palliative stenting for malignant dysphagia is associated with a technical success of approximately $95 \%$, a very low risk of early major complications (<5\%), and early clinical success of $80 \%$ [27]. Our study shows that without the use of fluoroscopy, both technical and clinical success is at par with the conventional technique.

Currently, palliative esophageal stenting is performed under fluoroscopic guidance and several studies have proven efficacy and safety [28-37]. However, there are several limitations of conventional fluoroscopic SEMS deployment. This requires demarcation of the proximal and distal extent of the stricture either with surface (skin) or inner markers. Surface markers, although easy to place and view, are often inaccurate because of parallax effects resulting from patient motion, including respiratory movements [38]. Additionally, the main advantage of using fluoroscopy is to allow passage of guidewire into the stomach and for placement of external radio-opaque markers at the two ends of the obstruction to allow accurate SEMS deployment. However, this leads to an increase in total procedure time and exposes patients to unnecessary radiation. Additionally, fluoroscopically guided insertions require additional equipment and personnel, and routine overnight stay adds an unnecessary additional cost to the service. Based on our study, we demonstrate that endoscopic placement of SEMS can be readily performed in medical centers that lack fluoroscopy. This is the first study to evaluate not only the feasibility but also the safety of this technique.

Based on our meta-regression analysis, the overall success did not appear to be affected by the tumor location within the esophagus or the length of obstruction. Meta-regression analysis, however, is a weak statistic in terms of assessing the predictive effects of a variable to the reported outcome. In 14 of the included studies [24, 25, 38-49], the esophageal stent was deployed under direct endoscopic view where as a through-thescope (TTS) technique was used in only one study [9]. In two studies $[50,51]$, the esophageal stent was placed over a guidewire and confirmation of accurate positioning was done by post deployment endoscopy.

There have been several AEs related to SEMS reported in literature, with incidence of stent migration ranging from $3 \%$ to $18 \%$ and that of tumor overgrowth, tumor tissue from progressive tumor growth or by nonmalignant hyperplastic tissue growth at the end of the stent, ranging from $2.5 \%$ to $10.5 \%$ [47]. Based on our analysis, the pooled incidence of stent migration was $4.1 \%$ and that of tumor overgrowth was $8.1 \%$, in concordance with the published literature. Clinically significant AEs were reported as early i.e. within 30 days post-procedure and late i.e. 30 days after the procedure. Most common early adverse event was retrosternal chest pain, reported in 185 patients $(10.4 \%)$ followed by gastro-esophageal reflux disease reported in 41 patients (2.3\%). Overall, there were 68 deaths (3.82\%) within 30 days of the procedure however these were not directly related to the procedure itself. When evaluating stent-related AEs, five patients had intra-procedural perforation related to stent insertion. Almond et al reported that of the three patients who had perforation during stent insertion, two underwent successful insertion of a covered esophageal wall stent, and neither required a repeat endoscopic, radiological, or surgical intervention. Both patients survived for more than 30 days following the procedure [39]. In another study by Kini et al, one patient each in two study groups, "with fluoroscopic guidance" and "without fluoroscopic guidance", respectively, had intra-procedural perforation [51]. Garcio-Cano reported mediastinitis in 1 patient due to a perforation during stent insertion which was closed with two other stents [25].

How does our study compare to other published reviews? Two prior studies have directly compared outcomes of endoscopic and fluoroscopic esophageal stenting. Kini et al directly compared outcomes of both these techniques with what they described as simplified technique involving blind placement of the esophageal stent over a guide wire. The authors concluded that both endoscopic and fluoroscopic techniques exhibited a comparable statistically significant improvement in dysphagia and that both techniques were equally safe. And while the conventional approach reduced procedure time and patient discomfort, the stents in the simplified technique were all fully covered and so a head-to-head comparison of techniques and outcomes could not be made with surety [51]. Ferriera et al concluded that both approaches are equally safe in terms of early and late complications [41]. To the best of our knowledge, no prior systematic reviews and meta-analysis have been reported on this topic.

The strengths of this review are as follows: systematic literature search with well-defined inclusion criteria, careful exclusion of redundant studies, inclusion of good quality studies with detailed extraction of data and rigorous evaluation of study quality. There are limitations to this study, most of which are inherent to any meta-analysis. The included studies were not entirely representative of the general population and community practice, with most studies being performed in tertiarycare referral centers, in the hands of expert endoscopists. Our analysis included studies that were retrospective in nature contributing to selection bias. We included studies where stenting was performed using various techniques and we were unable to assess if one method was superior to the other.

Our main aim was to evaluate the efficacy of esophageal stenting without the aid of fluoroscopy and for this reason, we included studies where an endoscopic, over-guidewire or through-the-scope techniques were used. We were unable to study the superiority and/ or inferiority of one technique over another. Considerable heterogeneity was observed based on the $12 \%$ values and the $95 \% \mathrm{PI}$ interval. Although we were unable to ascertain a statistical cause for the observed heterogeneity based on our meta-regression analysis, we believe the variability of the above mentioned techniques could explain the observed heterogeneity. Nevertheless, our study is the best available estimate in literature thus far with respect to the clinical outcomes of endoscopic palliative esophageal stenting. 


\section{Conclusion}

In conclusion, our meta-analysis demonstrates that palliative esophageal SEMS placement can be performed without the aid of fluoroscopy with a technical success rate of $94.7 \%$ and clinical success rate of $82.1 \%$, in expert hands and in high volume centers. To better establish its clinical role, future randomized controlled studies are needed comparing esophageal SEMS placement with fluoroscopy to without.

\section{Acknowledgements}

The authors thank Emily Glenn (Associate Director, Education \& Research Services, McGoogan Library of Medicine, University of Nebraska Medical Center, Omaha, Nebraska, United States) and Wade Lee-Smith (Research Engagement Librarian \& Science Reference Librarian, University of Toledo, Ohio, United States) for help with the systematic literature search.

\section{Competing interests}

The authors declare that they have no conflict of interest.

\section{References}

[1] Siegel RL, Miller KD, Jemal A et al. Cancer statistics, 2019. CA A Cancer J Clin 2019; 69: 7-34

[2] Arnold M, Soerjomataram I, Ferlay J et al. Global incidence of oesophageal cancer by histological subtype in 2012. Gut 2015; 64: 381387

[3] Pohl H, Sirovich B, Welch HG. Esophageal adenocarcinoma incidence: are we reaching the peak? Cancer Epidemiol Biomarkers Prev 2010; 19: $1468-1470$

[4] Engel LS, Chow W-H, Vaughan TL et al. Population attributable risks of esophageal and gastric cancers. J Natl Cancer Inst 2003; 95: 14041413

[5] Brierley JD, Oza AM. Radiation and chemotherapy in the management of malignant esophageal strictures. Gastrointest Endosc Clin N Am 1998; 8: 451-463

[6] Pennathur A, Gibson MK, Jobe BA et al. Oesophageal carcinoma. Lancet 2013; 381: 400-412

[7] Domschke W, Foerster EC, Matek W et al. Self-expanding mesh stent for esophageal cancer stenosis. Endoscopy 1990; 22: 134-136

[8] Eroglu A, Turkyilmaz M, Subasi M et al. The use of self-expandable metallic stents for palliative treatment of inoperable esophageal cancer. Dis Esophagus 2010; 23: 64-70

[9] Vermeulen BD, Reijm AN, van der Bogt RD et al. Through-the-scope placement of a fully covered metal stent for palliation of malignant dysphagia: a prospective cohort study (with video). Gastrointest Endosc 2019; 90: 972-979

[10] Moher D, Liberati A, Tetzlaff J et al. Preferred reporting items for systematic reviews and meta-analyses: The PRISMA statement. Annals Int Med 2009; 151: 264-269

[11] Stroup DF, Berlin JA, Morton SC et al. Meta-analysis of observational studies in epidemiology: a proposal for reporting. Meta-analysis Of Observational Studies in Epidemiology (MOOSE) group. JAMA 2000; 283: 2008-2012
[12] Stang A. Critical evaluation of the Newcastle-Ottawa scale for the assessment of the quality of nonrandomized studies in meta-analyses. Europ J Epidemiol 2010; 25: 603-605

[13] DerSimonian R, Laird N. Meta-analysis in clinical trials. Controlled Clin Trials 1986; 7: 177-188

[14] Sutton AJ, Abrams KR, Jones DR et al. Methods for Meta-Analysis in Medical Research. New York: John Wiley \& Sons Ltd; 2000: 205-228

[15] Higgins J, Thompson SG, Spiegelhalter DJ. A re-evaluation of randomeffects meta-analysis. J Royal Stat Soc 2009; 172: 137-159

[16] Riley RD, Higgins JP, Deeks JJ. Interpretation of random effects metaanalyses. BMJ 2011; 342: d549

[17] Mohan BP, Adler DG. Heterogeneity in systematic review and metaanalysis: how to read between the numbers. Gastrointest Endosc 2019; 89: 902-903

[18] Kanwal F, White D. Systematic Reviews and Meta-analyses in Clinical Gastroenterology and Hepatology. Clin Gastroenterol Hepatol 2012; 10: $1184-1186$

[19] Higgins JP, Thompson SG, Deeks J] et al. Measuring inconsistency in meta-analyses. BMJ 2003: 327-557

[20] Guyatt GH, Oxman AD, Kunz R et al. GRADE guidelines: 7. Rating the quality of evidence-inconsistency. J Clin Epidemiol 2011; 64: 12941302

[21] Easterbrook PJ, Gopalan R, Berlin JA et al. Publication bias in clinical research. Lancet 1991; 337: 867-872

[22] Duval S, Tweedie R. Trim and fill: a simple funnel-plot-based method of testing and adjusting for publication bias in meta-analysis. Biometrics 2000; 56: 455-463

[23] Rothstein HR, Sutton AJ, Borenstein M. Publication bias in meta-analysis: Prevention, assessment and adjustments. John Wiley \& Sons; 2006

[24] Austin AS, Khan Z, Cole AT et al. Placement of esophageal self-expanding metallic stents without fluoroscopy. Gastrointest Endosc 2001; 54: 357-359

[25] García-Cano J, Bermejo-Saiz E. Su1504 Endoscopic insertion of esophageal self-expanding metal stents to palliate malignant dysphagia without fluoroscopy. Gastrointest Endosc 2013; 77: AB349AB350

[26] Puhan MA, Schunemann H], Murad MH et al. A GRADE Working Group approach for rating the quality of treatment effect estimates from network meta-analysis. BMJ 2014; 349: g5630

[27] Anderloni A, Lollo G, Repici A. 28 - Palliation of malignant dysphagia and esophageal fistulas. Clin Gastrointest Endosc 2019: 316-321

[28] Adam A, Ellul J, Watkinson AF et al. Palliation of inoperable esophageal carcinoma: a prospective randomized trial of laser therapy and stent placement. Radiology 1997; 202: 344-348

[29] Christie NA, Buenaventura PO, Fernando HC et al. Results of expandable metal stents for malignant esophageal obstruction in $100 \mathrm{pa}-$ tients: short-term and long-term follow-up. Ann Thorac Surg 2001; 71: 1797-1801

[30] Cwikiel W, Tranberg KG, Cwikiel M et al. Malignant dysphagia: palliation with esophageal stents long-term results in 100 patients. Radiology 1998; 207: 513-518

[31] Dallal HJ, Smith GD, Grieve DC et al. A randomized trial of thermal ablative therapy versus expandable metal stents in the palliative treatment of patients with esophageal carcinoma. Gastrointest Endosc 2001; 54: 549-557

[32] Sabharwal T, Hamady MS, Chui S et al. A randomised prospective comparison of the Flamingo Wallstent and Ultra-flex stent for palliation of dysphagia associated with lower third oesophageal carcinoma. Gut 2003; 52: 922-926 
[33] Sarper A, Oz N, Cihangir C et al. The efficacy of self-expanding metal stents for palliation of malignant esophageal strictures and fistulas. Eur J Cardiothorac Surg 2003; 23: 794-798

[34] Siersema PD, Hop WC, van Blankenstein M et al. A comparison of 3 types of covered metal stents for the palliation of patients with dysphagia caused by esophagogastric carcinoma: a prospective, randomized study. Gastrointest Endosc 2001; 54: 145-153

[35] Verschuur EM, Homs MY, Steyerberg EW et al. A new esophageal stent design (Niti-S stent) for the prevention of migration: a prospective study in 42 patients. Gastrointest Endosc 2006; 63: 134-140

[36] Xinopoulos D, Dimitroulopoulos D, Moschandrea I et al. Natural course of inoperable esophageal cancer treated with metallic expandable stents: quality of life and cost-effectiveness analysis. J Gastroenterol Hepatol 2004; 19: 1397-1402

[37] Yajima K, Kanda T, Nakagawa S et al. Self-expandable metallic stents for palliation of malignant esophageal obstruction: special reference to quality of life and survival of patients. Dis Esophagus 2004; 17: 7175

[38] Lazaraki G, Katsinelos P, Nakos A et al. Malignant esophageal dysphagia palliation using insertion of a covered Ultraflex stent without fluoroscopy: a prospective observational study. Surg Endosc 2011; 25: 628

[39] Almond LM, Patel K, Keast L et al. A decade of day-case endoscopically guided stent placement in malignant oesophagogastric strictures. Surg Endosc 2017; 31: 2280-2286

[40] Ben Soussan E, Antonietti M, Lecleire $S$ et al. Palliative esophageal stent placement using endoscopic guidance without fluoroscopy. Gastroenterol Clin Biol 2005; 29: 785-788

[41] Ferreira F, Bastos P, Ribeiro A et al. A comparative study between fluoroscopic and endoscopic guidance in palliative esophageal stent placement. Dis Esophagus 2012; 25: 608-613
[42] Govender M, Aldous C, Ferndale L et al. Self-expanding metal stent placement for oesophageal cancer without fluoroscopy is safe and effective. S Afr Med J 2015; 105: 858-861

[43] Kumar JP, Babu GR. Safety of endoscopic self-expanding metallic stent placement in esophageal cancer without fluoroscopy. Int Surgery J 2016; 3: 1757-1760

[44] Saligram S, Lim D, Pena L et al. Safety and feasibility of esophageal self- expandable metal stent placement without the aid of fluoroscopy. Dis Esophagus 2017; 30: 1-6

[45] Sharma V, Maru A, Mangal D. Palliative esophageal metal stenting under endoscopic guidance without the use of fluoroscopy: initial experience at an indian centre. Annals Oncology 2012; 23: iv31-iv118

[46] Siddiqui AA, Ansari S, Ghouri MA et al. Self expandable metallic stent endoscopic insertion in esophageal cancer, J Coll Physicians Surg Pak. 2010; 20: 502-505

[47] Tahiri M, Ferraro P, Duranceau A et al. Self-expanding metallic stent placement with an exaggerated $5-\mathrm{cm}$ proximal tumor covering for palliation of esophageal cancer. Annals Gastroenterol 2015; 28: $347-$ 352

[48] White RE, Mungatana C, Topazian M et al. Esophageal stent placement without fluoroscopy. Gastrointest Endosc 2001; 53: 348-351

[49] Wilkes EA, Jackson LM, Cole AT et al. Insertion of expandable metallic stents in esophageal cancer without fluoroscopy is safe and effective: a 5-year experience. Gastrointest Endosc 2007; 65: 923-929

[50] Balekuduru AB, Sahu MK, Sreenivasa KKA et al. Efficacy and safety of endoscopic self-expanding metallic stent for esophageal malignancy: a two-institute experience. J Digest Endosc 2019; 10: 101-106

[51] Kini R, Ramanathan S, Thangavel P et al. A simplified technique of esophageal self-expandable metallic stent placement without fluoroscopic and endoscopic guidance for treating esophageal carcinoma. Turk J Gastroenterol 2018; 29: 82-88 